\title{
Interference Reduction by Null-forming Optimization Based MVDR Technique Using Gravitational Search Algorithm
}

\author{
Suhail Najm Shahab ${ }^{1 *}$, Ayib Rosdi Zainun ${ }^{1}$, Waleed M. Sh. Alabdraba ${ }^{2}$, Nurul \\ Hazlina Noordin ${ }^{1}$ and Izzeldin Ibrahim Mohamed $^{1}$ \\ ${ }^{1}$ Faculty of Electrical and Electronics Engineering, Universiti Malaysia Pahang, 26600, \\ Pahang, Malaysia \\ ${ }^{2}$ Environmental Engineering Department, College of Engineering, Tikrit University, Tikrit, \\ Iraq. \\ \{68suhel,ayib,hazilna,izzeldin, walabdraba\}@gmail.com, ump.edu.my, tu.edu.iq
}

\begin{abstract}
Interference reduction one of the major problem in the wireless communication systems. Minimum Variance Distortionless Response (MVDR) Beamformer is the process of extracting the user-of-interest (UOI) signal from interferer signals and external noise. In the present work, Gravitational Search Algorithm (GSA) is a new modern metaheuristic optimization technique used for optimizing the MVDR null-forming level by controlling the excitation weight coefficients in a linear antenna array radiation pattern synthesis. The best set of complex excitation coefficients solution in multidimensional problems evaluated based on two figure of merit, signal-to-interference-plus-noise ratio (SINR) and beampattern accuracy for two scan angles. The results reported here have been compared with the results of conventional MVDR technique. MVDR ${ }_{G S A}$ successfully used to calculate the excitation weight coefficients with the desired patterns for both accurate beam shaping and introduce deep pattern nulling. It found that the SINR and output beampattern were in good agreement with MVDRGSA.
\end{abstract}

Keywords: Beamforming; Linear Antenna Array; Minimum Variance Distortionless Response, SINR, Smart Antenna.

\section{Introduction}

Beamforming (BF) algorithms have gained wide attention by researcher's community due to the wider range of application. MVDR or Capon beamformer [1] is one of the optimum statistical beamformers which assures a distortionless response for a predefined steering direction [2-4]. The basic idea of the MVDR technique is to estimate the excitation coefficients in an adaptive manner by minimizing the variance of the residual interference and noise while enforcing a set of linear constraints to ensure that the real user signal is not distorted [5]. MVDR weight vector solution depends on the array response vector and the estimation of the covariance matrix of user-of-interest (UOI) signals and user-not-of-interest (UNOI) sources. The null-forming for MVDR has poor SINR output due to low null-forming level towards the UNOI signals when multiple access interference is existing [6-8], the finite size of data snapshots $[9,10]$ or the array response vector uncertainty [11]. 
There are many ways to make the MVDR beamformer robust against this error such as diagonal loading [12] or beamspace processing [13]. This empirical framewo-rk does not always lead to a solution that is easily identifiable. Therefore, optimizatio-n methods can be applied to provide a robust solution for the SASs. Some researchers have presented numerical techniques involving nature-inspired optimization to impro-ve the antenna beampattern, beamwidth, sidelobe control, phase shifter, or complex weight vector based conventional beamforming techniques [6, 8, 14-16]. A study in [8] combines the Linear Constrain Minimum Variance (LCMV) beamformer techniq-ue with particle swarm optimization (PSO), dynamic mutated-artificial immune system (DM-AIS), and gravitational search algorithm (GSA) to improve the complex excitation coefficients of LCMV beamforming technique. The most effective solution founded by GSA algorithm among other for all simulation results. Similarly, [17] proposed a phase-only pattern optimization by using GSA based on concentric ring antenna array of reconfigurable dual-beam. Another study is done to combine GSA with direction of arrival (DOA) method based antenna array system for enhancing the accuracy of DOA estimation of the incident angles [18]. Chatterjee and co-authors applied GSA and modified PSO to reduce the sidelobe effects in the antenna beampattern. Among these studies, GSA gives a superior performance or at least comparable improvement than others. In [6] the authors combined two intelligent swarm algorithms to improve the MVDR weight vector. Unfortunately, it does not obviously state the noise power of the received signals, because the noise power has a significant impact on the MVDR nullforming $[19,20]$. On the other hand, the effects of population size and a number of maximum iteration also not explicitly mentioned and investigated. Thus, the solution of this study is not the most accurate one.

In this study, the null-forming of the MVDR technique is enhanced using GSA. GSA is one of the modern heuristic optimization algorithm applied to the smart anten-na system. The performance evaluation for the comparison purpose is based on two figure of merit, SINR and beampattern accuracy for two scan angles which are still unknown from the expressions. The null width in the azimuth and elevation scanning angle also have been assessed. The weights excitation coefficients calculated to place deep and sharp nulls toward the UNOI direction accurately, and unity gain response toward the direction of UOI. Simulation results confirm the accuracy of the numerical results. Also, a GSA has an excellent agreement to solve antenna array problems by controlling the complex weight vector solution. The outline of this work is organized as follows: section 2, proposed approach along with the фonventional MVDR system model are described. Section 3, highlight the significant outcomes from MVDR based GSA (MVDR ${ }_{\mathrm{GSA}}$ ) combinations. Lastly, the conclusion is given in section 4.

\section{MVDR Beamformer Design Model}

The basic theory of the BF and the signal structure is presented in this section. The signal model considers $L$ sources incident on a uniform linear array (ULA) of $M$ isotropic elements, and the spacing between neighboring antennas is a half of wavelength. Assume that $L$ signal is coming from angles of $\theta_{l}$ and $\phi_{l}$ is incident upon an antenna array of $M$ elements. Here, the impinging angles of $\theta$ and $\phi$ are the 
azimuthal and elevation angles, respectively. The received signal, $r_{m}(k) \in \mathbb{C}^{\mathrm{M} \times \mathrm{K}}$, at the $m^{\text {th }}$ antenna in the $k^{\text {th }}$ snapshot incident upon the antenna array can be written as:

$$
r_{m}(k)=\sum_{s=1}^{S} x_{s}(k) \hat{a}_{s}(\theta, \phi)+\sum_{i=1}^{I} x_{i}(k) \hat{a}_{\mathrm{i}}(\theta, \phi)+\sum_{m=0}^{M-1} n_{m}(k)
$$

Where $x_{s}(k), x_{i}(k)$, and $n_{m}(k)$ denote the $s^{\text {th }}$ UOI signals, $i^{\text {th }}$ interference signals and additive background White Gaussian noise at the $m^{\text {th }}$ elements, respectively. Among these $L$ incident signals, it is assumed that $x_{s}(k)$ is the desired UOI and $x_{i}(k)+n_{m}(k)$ are the UNOI signals. The array response vector, $\hat{\mathrm{a}}\left(\theta_{l}, \phi_{l}\right) \in \mathbb{C}^{\mathrm{M} \times 1}$ of a ULA with $M$-elements where $\left(\theta_{l}, \phi_{l}\right)$ is the DOAs of the $l^{\text {th }}$ signal component calculated as [4]:

$$
\widehat{a}(\theta, \phi)=\left[1, e^{-j \beta \delta \sin \theta \sin \phi}, \ldots, e^{-j(M-1) \beta \delta \sin \theta \sin \phi}\right]^{*}
$$

where $\beta=2 \pi / \lambda$ is the free-space wavenumber, $\delta$ is the separation between two elements and $\lambda$ is the free-space wavelength. The $\theta \in[-\pi / 2, \pi / 2], \phi \in[0, \pi / 2]$ and (.) $)^{*}$ denote the complex conjugate. The $\hat{\mathrm{a}}\left(\theta_{l}, \phi_{l}\right)$ is a function of the incident angles, the location of the antenna, and the array geometry. It plays an important role in smart antenna systems, containing information of the impinging angles. The output of the beamformer at the $k^{\text {th }}$ snapshots, $\mathrm{y}(k)$ after signal processing is defined as:

$$
y(k)=\sum_{m=0}^{M-1} h_{m}^{\dagger} r_{m}(k)
$$

where $h$ is a complex multiplicative weight vector given as $\left[h_{0}, h_{1}, \ldots, h_{m}, h_{M-1}\right]^{\mathrm{T}}$ multiplied by the received signal at the $m^{\text {th }}$ antenna element and $(.)^{\dagger},(.)^{\mathrm{T}}$ denotes respectively the complex conjugate transpose and Hermitian transpose of a vector or matrix. The array covariance matrix $\Gamma_{r} \in \mathbb{C}^{\mathrm{M} \times \mathrm{M}}$ is the second-order statistical property of the impinging signals. In real applications, $\boldsymbol{\Gamma}_{r}$ is estimated using the received array snapshots. The estimated array covariance matrix is defined as [3]:

$$
\hat{\boldsymbol{\Gamma}}_{r} \cong \frac{1}{K} \sum_{k=1}^{K} r_{m}(k) r_{m}^{\dagger}(k) \cong \underbrace{\sum_{s=1}^{S} \sigma_{s}^{2} \hat{a}_{s}(\theta, \phi) \hat{a}_{s}^{\dagger}(\theta, \phi)}_{\boldsymbol{\Gamma}_{s}}+\underbrace{\sum_{i=1}^{I} \sigma_{i}^{2} \hat{a}_{i}(\theta, \phi) \hat{a}_{i}^{\dagger}(\theta, \phi)+\sigma_{n}^{2} \Lambda_{m}}_{\boldsymbol{\Gamma}_{i+n}}
$$

Where $K$ is the number of available snapshots. $\boldsymbol{\Gamma}_{s}$ and $\boldsymbol{\Gamma}_{i+n}$ denote the array correlation matrix corresponding to the desired UOI and the undesired UNOI, respectively. The

terms $\sigma_{s}^{2}, \sigma_{i}^{2}$ and $\sigma_{n}^{2}$ denotes the real user, interference, and noise powers. $\Lambda_{m} \in \mathbb{R}^{\mathrm{M} \times \mathrm{M}}$ stands for the identity matrix. It is known from the literature that the optimization criterion for MVDR [1] forms weights in a way that will attempt to maintain unity

gain of the beamformer in the beam angle direction while steering nulls in the direction of interference [21]. The weights are calculated by solving the following minimization equations with unity gain restraint:

$$
h_{M V D R}=\arg \min _{h} \mathrm{E}\left\{|\mathrm{y}(\mathrm{k})|^{2}\right\} \Rightarrow \min h^{\dagger} \hat{\boldsymbol{\Gamma}}_{r} h \text { s.t. } h^{\dagger} \widehat{a}_{s}(\theta, \phi)=1
$$

The Lagrange multipliers are used to solve MVDR constrain, resulted in weight vector calculated as follows [22]:

$$
h_{M V D R}=\widehat{a}(\theta, \phi) \hat{\boldsymbol{\Gamma}}_{r}^{-1}\left[\hat{a}^{\dagger}(\theta, \phi) \hat{\boldsymbol{\Gamma}}_{r}^{-1} \hat{a}(\theta, \phi)\right]^{-1}
$$

Antenna radiation patterns are typically expressed in terms of radiated power. The output power is defined as [4]:

$$
P_{y}=E\left\{y(k) y^{\dagger}(k)\right\}=h^{\dagger} E\left\{y(k) y^{\dagger}(k)\right\} h=h^{\dagger} \hat{\boldsymbol{\Gamma}}_{r} h
$$

Equation (7) can be rewritten as: 


$$
P_{y}=h^{\dagger} \boldsymbol{\Gamma}_{s} h+h^{\dagger} \boldsymbol{\Gamma}_{i+n} h=\underbrace{\sum_{=1} \sigma_{s}^{2}\left|h^{\dagger} \hat{a}_{s}(\theta, \phi)\right|}_{P_{s}}+\underbrace{{ }_{i=}^{I} \sigma_{i}^{2}\left|h^{\dagger} \hat{a}_{i}(\theta, \phi)\right|+\sigma_{n}^{2}}_{P_{i+n}}
$$

Where the $P_{s}$ denote the power of the desired signal and $P_{i+n}$ refer to the power output in the direction of UNOI. Finally, the SINR is defined as the ratio of the average power of the desired signal divided by the average power of the undesired signal computed as [23]:

$$
S I N R \triangleq \frac{P_{s}}{P_{i+n}} \triangleq \frac{\sum_{s=1}^{S} \sigma_{s}^{2}|h \quad \hat{a}(\theta, \phi)|}{\sum_{i=1}^{I} \sigma_{i}^{2}\left|h^{\dagger} \hat{a}_{i}(\theta, \phi)\right|+\sigma_{n}^{2}}
$$

\subsection{Gravitational search algorithm (GSA)}

Metaheuristic approaches have drawn considerable attention from many researchers

in the past and still of great interest. GSA is one of the most popular metaheuristics methods, proposed by Rashedi and his colleagues [24] based on heuristic optimization inspired by Newton's laws of motion and gravity. The present section introduces a po-

pulation-based search algorithm based on the law of gravity and mass interaction. All the force of gravity attracted objects with each other, and that causes a global movem-

ent towards the heavier mass object. Therefore, they use the direct form of communic-ation objects through gravitational forces. According to the best solution with heavier masses, which move more slowly than the lighter that guarantees the step of using this

algorithm to find a better solution. Each agent in GSA has four specifications: position, inertial mass, active gravitational mass and passive gravitational mass. The position of the mass is suitable for solving the problem, and it is gravitational, and

inertial masses decides to use an appropriate function. This mass will show an optimal solution in the search space. To optimize such a problem, it is necessary to determine the search space. These solutions are defined in a particular search space to generate

the $i^{\text {th }}$ initial position of the $N$ number of the individual agent randomly in the search

space. Every possible solution is a mass for the GSA. The system is designed in this section will consist of several masses. The position of the masses as below:

$$
x_{i} \quad\left(x_{i}^{1}, \ldots, x_{i}, \ldots, x_{i}^{n}\right) ; i=1,2, \ldots, N
$$

Where $x_{i}^{d}$ denote the position of the individual masses at the $i^{\text {th }}$ agent in the $d^{\text {th }}$ dimension of $N$ space dimension. At each generation, the best and worst of the calculated fitness value is selected for all agents and the improvements are made to maximize the problem defined as:

$$
\begin{aligned}
& \operatorname{best}(t)=\max _{j \in\{1, \ldots,}, f_{i t_{j}(t)} \\
& \operatorname{worst}(t)=\min _{j \in\{1, \ldots,} f f_{j}(t)
\end{aligned}
$$

Where $\operatorname{best}(t)$ and $\operatorname{worst}(t)$ is expressing the best and worst solutions in the iteration $t$, fit $t_{j}(t)$ shows the fitness value and the suitability of individual $j$ at moment $t$. Each mass is calculated with the current fitness value of the fitness function. The gravitational constant $G$, will be reduced exponentially in every generation, and is initially set to 
control the search accuracy. In other words, $G$ is the function of the initial value $\left(G_{0}\right)$ and $t$, computed as follows:

$$
G(t)=G_{0} \times e^{-\alpha\left(t / t_{\max }\right)}
$$

Where, $G_{0}$ is the initial value of the gravitational constant, $\alpha$ a fixed value that the user determines, and $t_{\max }$ is the total number of iterations generations. The active mass in a gravitational mass of a search space based on passive gravitational mass and inertial mass by taking all masses equal to each other, the updated weight is calculated when evaluating the fitness through the following equation:

$$
\begin{gathered}
M_{a i}=M_{p i}=M_{i i}=M_{i} ; \quad i=1,2, \ldots, N \\
M_{i}(t)=m_{i}(t) / \sum_{j=1}^{N} m_{j}(t) \\
m_{i}=\operatorname{fit}(t)-\operatorname{worst}(t) / \operatorname{best}(t)-\operatorname{worst}(t)
\end{gathered}
$$

Where $M_{a i}, M_{p i}, M_{i i}, M_{i}$ denotes respectively, the active gravitational mass of bodies, passive gravitational mass, inertia mass and $i^{\text {th }}$ individual inertial mass. In Equation above normalization process is carried out, the heavy mass as determined by the mass update is the most effective. It moves slower than others that are more effective mass in the search space and attracts others better [17]. Then, by the laws of motion, the acceleration, $a_{i}{ }^{d}(t)$, of the individual $i$ at $t^{t h}$ generation in $d^{t h}$ dimension can be computed as:

$$
a_{i}^{d}(t)=F_{i}^{d}(t) / M_{i i}(t)
$$

Where $F_{i}^{d}(t)$ is the total force acting on a mass $i$ is calculated after calculating the force between two masses:

$$
F_{i}(t)=\sum_{\substack{j \in N \text { Nbest } \\ j \neq i}} \text { rand }_{j} F_{i j}^{d}(t)
$$

Where rand $_{j}$ it is a random number in the range $[0,1]^{d}$, Nbest is the initial starting value and function decreasing with time in a certain way that includes the best fitness value to prevent discovery initially remain in local optimum and $F_{i j}{ }^{d}(t)$ is the forces between two bodies that acting on the mass $i$ from $j$ is defined as:

$$
F_{i j}^{d}(t)=G(t)\left[\left(M_{p i}(t) \times M_{a j}(t)\right) /\left(R_{i j}(t)+\varepsilon\right)\right]\left(x_{j}^{d}(t)-x_{i}^{d}(t)\right)
$$

Where $M_{a j}$ associated with thej individual active gravitational mass, $M_{p i}$ associated with thei individuals passive gravitational mass, $G(t)$ calculated at the same time, $t, \varepsilon$ is a numerically small constant decided by a user. $x_{j}^{d}(\mathrm{t}), x_{j}{ }^{d}(\mathrm{t})$ refer to $i$ and $j$ masses and $R_{i j}(\mathrm{t})$, the Euclidean distance between the two point masses in the search space ( $i$ and $j$ members). All masses are accelerated in the search space interact with each other. Further, a next speed of the agent is considered part of the current speed state attached to its speed. Therefore, the new velocity and position at next iteration along the $d^{\text {th }}$ dimension can be upgraded as follows:

$$
\begin{aligned}
& v_{i}^{d}(t+1)=\operatorname{rand}_{i} \times v_{i}^{d}(t)+a_{i}^{d}(t) \\
& x_{i}^{d}(t+1)=x_{i}^{d}(t)+v_{i}^{d}(t+1)
\end{aligned}
$$

Where $v_{i}^{d}(t)$ and $x_{i}^{d}(t)$ represents the velocity and the position of $i^{t h}$ agents in the $d^{\text {th }}$ dimension at the $t^{\text {th }}$ iteration, respectively. $\operatorname{rand}_{i}$ denote random values in the interval of $[0,1]^{d}$. By gravitational and inertial mass conformity assessment calculated in a simple manner. A heavier weight means more effective than an individual. It is better to have a greater attraction of the individual and is not meant slower moving it. The 
inertial mass and gravitational mass assuming equality values conformity of mass mapping are calculated. The details on how to combine conventional MVDR with a numerical technique involving nature-inspired optimization is described in next section for using the weighted objective approach.

\subsection{Problem description and formulation}

The null-forming is an important process in the modern communication systems for maximizing SINR. The most common MVDR problem is that the signal model must be entirely accurate in order not to form unity gain in the UOI direction nulls in the direction of the UNOI. When the size of data snapshots is small will result in a poorly represented beampattern and degrades the MVDR performance. However, null-forming of the MVDR affected by these errors. Therefore, the task of combine the conventional MVDR $\left(\mathrm{MVDR}_{\mathrm{con}}\right)$ with nature-inspired metaheuristic method is to find appropriate complex excitation coefficient that minimizing $P_{i+n}$ by deep null-forming and hence high SINR can be obtained. On the other hand, desired user power is satisfying the MVDR constrain. Firstly, a population of agents is initialized with random position except for the first set of agents replaced by the weight vector obtained from $\mathrm{MVDR}_{\mathrm{con}}$ in the search space dimension and these position vector at specified dimension is converted to a candidate solution vector to this problem as shown in Equation 10. Afterward, the fitness function evaluated in each iteration to find SINR $\left.\right|_{\max }$ by minimizing power given to reach the UNOIs directions.

$$
\left.h_{m}^{\dagger}=x^{d}(t) \quad \begin{array}{cccc}
h_{0} & h_{1} & \cdots & h_{M-1} \\
x_{1}^{1}(t+1) & x_{1}(t+1) & \cdots & x_{1}^{d}(t+1) \\
\vdots & \vdots & \ddots & \vdots \\
x_{N}^{1}\left(t_{\max }\right) & x_{N}\left(t_{\max }\right) & \cdots & x_{N}^{d}\left(t_{\max }\right)
\end{array}\right]
$$

Where $\sum_{m=0}^{M-1} h_{m}^{\dagger} \in \mathbb{C}^{\mathrm{M} \times 1}$ is analogues to the $x_{N}{ }^{d}$, where $N$ is the best solution for the total population size in each iteration with the number of variable in the search space dimension equal to the number of elements $(M=d)$. In the next iteration, each agent calculates a new velocity and a new position according to the Eqs. 19 and 20. In order to have a tradeoff between the antenna array pattern synthesis and the optimization method, the fitness function, $f f$ can be calculated using the following equation:

$$
f f=\arg \max _{x_{N}^{d}}\left[P_{s} / P_{i+n}\right] \text { s.t. } \min P_{i} n
$$

Where $P_{S}$ denote the power of the desired user and $P_{i+n}$ refer to the interference and noise power. GSA is used to obtain the radiation pattern with SINR $\left.\right|_{\max }$. Termination criteria fixed by updating the algorithm until iteration reaches their maximum limit.

Then return the best-so-far fitness value at the final iteration as the global fitness of the problem and the positions of the corresponding agent at specified dimensions as the global solution of that problem. 


\section{Simulation and Discussion Results}

This section presents a three simulation experiments performed to demonstrate the performance of the $\mathrm{MVDR}_{\text {con }}$ and the $\mathrm{MVDR}_{\mathrm{GSA}}$. A set of parameters that control GSA behavior when optimizing a given problem is shown in Table 1 as well as the MVDR beamformer simulation parameters setting, where the proposed algorithm is executed 20 times, and the best results are recorded. It is worth noting that the GSA parameters are taken from [24] as standard ones. The total received signal power assumed to be $\mathrm{SNR}=\mathrm{INR}$ of $10 \mathrm{~dB}$. The weight vector of each element depended on the incident angle and array covariance matrix estimation. The weight vector for $\mathrm{MVDR}_{\text {con }}$ is calculated using Eq. 6. In order to obtain the weights needed, Eq. 21 will be utilized by the intelligent swarm optimization process to achieving SINR $\left.\right|_{\max }$.

Table 1. Key intrinsic parameters for MVDR and GSA

\begin{tabular}{lclc}
\hline Key system parameters & Values & Key system parameters & Values \\
\hline Array antenna geometry & ULA & Population size $(N)$ & 10 \\
Antenna type & Isotropic & Search space dimension $(d)$ & 4,8 \\
Carrier frequency $(f c)$ & $2.6 \mathrm{GHz}[12]$ & Termination condition $\left(t_{\max }\right)$ & 500 \\
Beam scanning $\left[\theta^{\circ}, \phi^{\circ}\right]$ & {$[ \pm 90,0: 90]$} & Initial Grav. constant $\left(G_{0}\right)$ & $100[24]$ \\
Number of elements $(M)$ & 4,8 & Gradient constant $(\alpha)$ & $20[24]$ \\
Element spacing $(\delta)$ & $\lambda / 2$ & Zero offset constant $(\varepsilon)$ & $2.2 \mathrm{e}-16[24]$ \\
Snapshots $(K)$ & 250 & Random interval & rand $[0,1]^{d}$ \\
SNR [dB] $=\operatorname{INR}[\mathrm{dB}]$ & 10 & ff limit & Max \\
UOI direction $\left[\theta_{s}^{\circ}, \phi_{s}^{\circ}\right]$ & 0,0 & Null-forming limit & Min \\
UNOI direction $\left[\theta_{i}^{\circ}, \phi_{i}^{\circ}\right]$ & $\pm 15,0- \pm 30,0$ & & \\
\hline
\end{tabular}

The first experiment consists in simulating a 4-element linear antenna array with one desired transmitter and two interferers. Both the $\mathrm{MVDR}_{\text {con }}$ and the $\mathrm{MVDR}_{\mathrm{GSA}}$ al-gorithms will obtain an optimum weight vector that configures the array in such a way that the resulting mainlobe points towards the desired signal while the array patt-ern presents a null in the direction of the interferers. The aim of the experiment is to com-pare the results obtained by the algorithms concerning average null-forming that $\left.\mathrm{SINR}\right|_{\max }$ can be achieved. Uniform linear array with element inter-spacing of $0.5 \lambda$ is considered and 500 iterations are performed. The UOI has an AOA at $\theta_{s}=0^{\circ}$ and two UNOIs at $\pm 30^{\circ}$ while the elevation angles are fixed as $\phi_{s}=\phi_{i}=0^{\circ}$. Fig. 1(a) shows the convergence rate of the MVDR $_{\mathrm{GSA}}$ when optimizing a 4-element using SINR as ff. The graph indicates that the best-so-far and mean SINR $\left.\right|_{\max }$ of MVDR $_{\mathrm{GSA}}$ method is obtained 161.4 $\mathrm{dB}, 149.2 \mathrm{~dB}$ compared to $41.3 \mathrm{~dB}$ for $\mathrm{MVDR}_{\mathrm{GSA}}$ and $\mathrm{MVDR}_{\text {con }}$, resp-ectively. It is clearly seen that $\mathrm{MVDR}_{\mathrm{GSA}}$ provide significant $f f$ improvement with keep changing, and this means obvious local minima. Fig. 1(b) shows the normalized beampattern for both techniques for four isotropic elements. For this problem, $d=4$ which is the dimension of the problem and the result consists of an excitation weight coefficient of $\mathbb{C}^{4 \times 1}$, each one corresponding to one element. It can be seen that the $\mathrm{MVDR}_{\mathrm{GSA}}$ algorithm obtains the lowest null levels compared with the $\mathrm{MVDR}_{\text {con }}$ technique, the average null observed is $-40.5 \mathrm{~dB}$ and $-161.0 \mathrm{~dB}$ for MVDRcon and MVDR $\mathrm{GSA}_{\text {, }}$ respectively giving $\mathrm{a} \approx 297 \%$ null improvements over $\mathrm{MVDR}_{\mathrm{con}}$. These results show that $\mathrm{MVDR}_{\mathrm{GSA}}$ algorithm is capable of obtaining the desired results inde- 
pendently within a small number of array elements and places a perfect null at each interference source direction. Figs 1(c), (d), (f) and (g) shows the 3D beampattern for azimuthal, $\theta^{\circ}$ and elevation, $\phi^{\circ}$ scan angles plots of the $\mathrm{MVDR}_{\text {con }}$ and $\mathrm{MVDR}_{\mathrm{GSA}}$, respectively. The power is measured in $\mathrm{dB}$ and the color bar is used for a sense of the relative scale of the power. It can be easily seen by comparing these figures, the null width in the $\theta^{\circ}$ and $\phi^{\circ}$ obtained by $\mathrm{MVDR}_{\mathrm{GSA}}$ narrower than $\mathrm{MVDR}_{\mathrm{con}}$ in addition to sharp null-forming. It is observed that the $\mathrm{MVDR}_{\mathrm{GSA}}$ give very deepest null-forming with a null width of almost $3^{\circ}$ compared to $10^{\circ}$ by $\mathrm{MVDR}_{\text {con }}$, respectively. The $\mathrm{MVDR}_{\text {con }}$ mainbeam accuracy is skewed by $2^{\circ}$ from the target direction while $\mathrm{MVDR}_{\mathrm{GSA}}$ provides accurately mainbeam. The corresponding best final solution (complex excitation weight) obtained from $M_{V D R}$ con and $M_{V D R}{ }_{G S A}$ are shown in Fig 1(e)-(h) that is represents the amplitude and phase $(A \forall \Phi)$ of each antenna element.

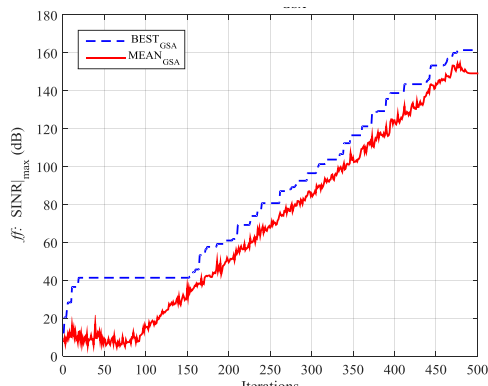

(a)

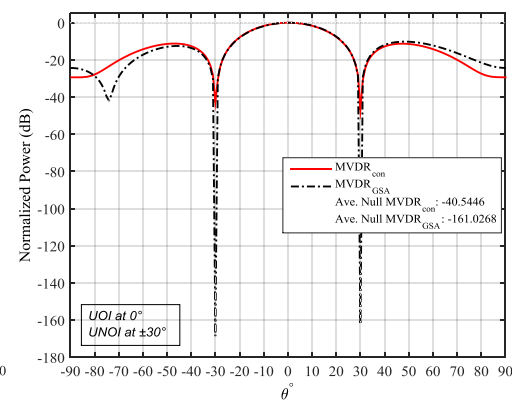

(b)

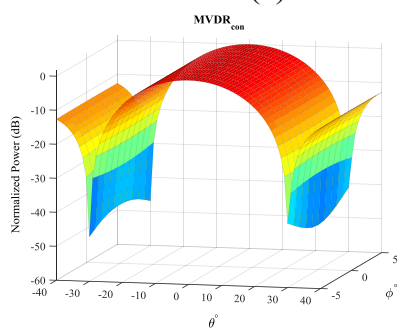

(c)

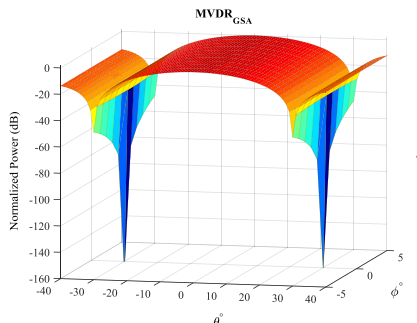

(f)

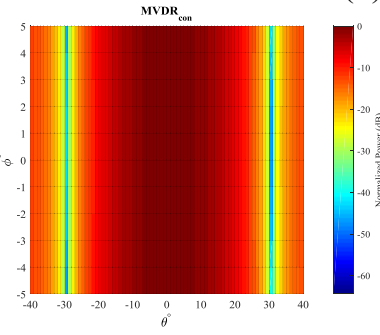

(d)

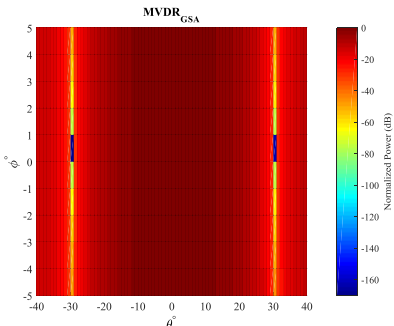

(g)

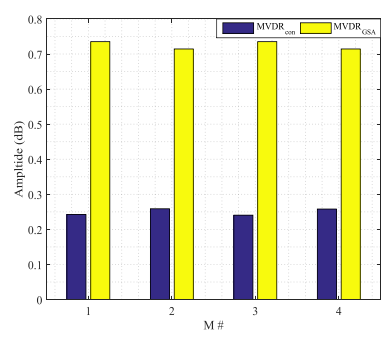

(e)

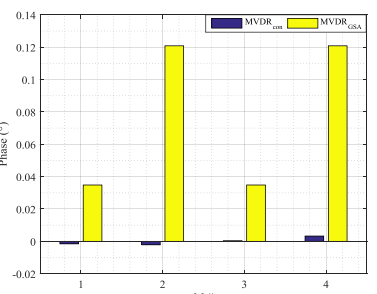

(h)

Fig. 1. Comparison of SINR $\left.\right|_{\max } \mathrm{MVDR}_{\text {con }}$ vs. MVDR $\mathrm{GSA}$; UOI at $0^{\circ}$, UNOIs at $\pm 15^{\circ}, M=4$, $t_{\max }=500, N=10$; (a) Best and mean SINR. (b) Normalized beampattern. (c)-(g) 3D beampattern in term of azimuth and elevation scan angles. (e)-(h) complex weights vector. 
The second experiment is similar to the first experiment and the aim is to test both algorithms to a more difficult environment, namely having four undesired signals instead of two with search dimension increased to 8 . Given the difficulty of obtaining a suitable array pattern, the second experiment is performed for an 8-element linear antenna array is defined. The desired user angle at $0^{\circ}$ and a number of four undesired directions are set at $\pm 15^{\circ}$ and $\pm 30^{\circ}$, respectively.

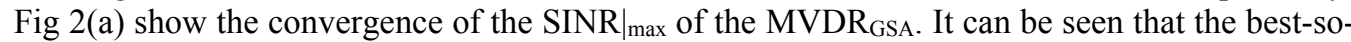
far and mean values are obtained of $500^{\text {th }}$ iterations based 10-population size around $147.6 \mathrm{~dB}$ and $146.5 \mathrm{~dB}$, respectively comparing to the $\left.\mathrm{SINR}\right|_{\max }$ obtained by $\mathrm{MVDR}_{\text {con }}$ is $50.3 \mathrm{~dB}$. Fig 2(b) shows an illustration of the resulting radiation pattern. It is clear that $\mathrm{MVDR}_{\text {con }}$ managed to get nulls at the UNOIs direction, but the proposed $\mathrm{MVDR}_{\mathrm{GSA}}$ produces a sophisticated solution that is placing deep null-forming with average null found to be $-45.6 \mathrm{~dB}$ and $-147.4 \mathrm{~dB}$, respectively and the percentage of null-forming improvement $\approx 223 \%$. Also, it can clearly be seen that the mainlobe of the radiation patterns are directed toward the desired angles of UOI $\left(\theta_{s}=0^{\circ}, \phi_{s}=0^{\circ}\right)$ in both methods. Moreover, the width of the mainlobe decreases as the number of array elements is increased; in other words, it becomes narrower and high directivity. Figs 2(c), (d), (f) and (g) are graphs that show the behavior of MVDR width and sharpness in the $\theta^{\circ}$ and $\phi^{\circ}$. It can be seen that the MVDR $\mathrm{GSA}_{\text {a }}$ null-width position in

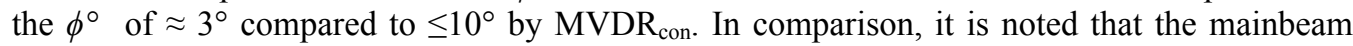
width of the 4-element array is the widest while the 8-element array is the narrowest. Therefore, the width of the mainlobe decreases as the number of array elements is increased; in other words, it becomes narrower and high directivity. The required complex excitation of each element that resulting radiation pattern of SINR $\left.\right|_{\max }$ is shown in Figs 2(e)-(h).

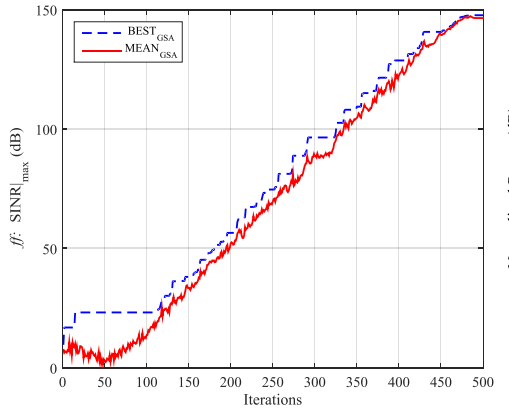

(a)

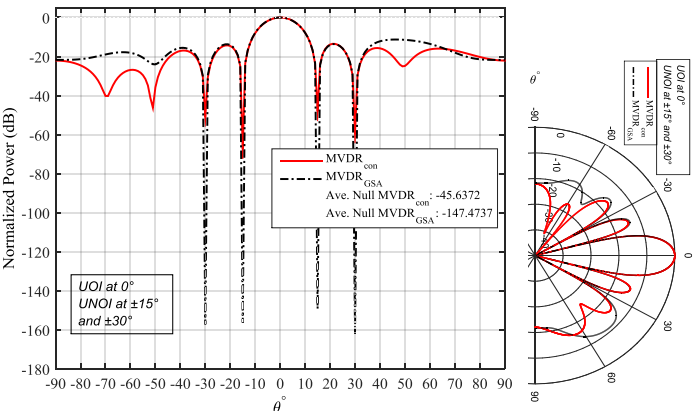

(b)

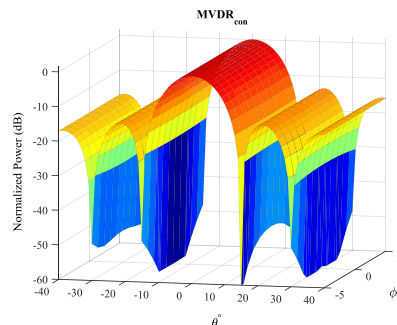

(c)

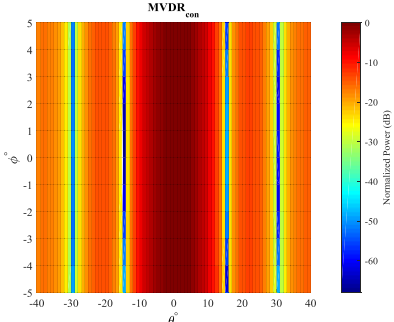

(d)

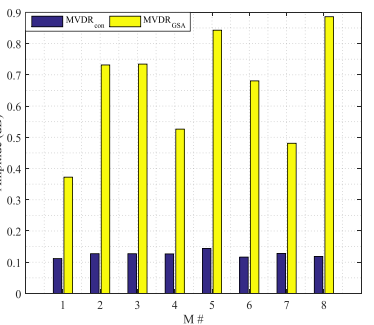

(e) 


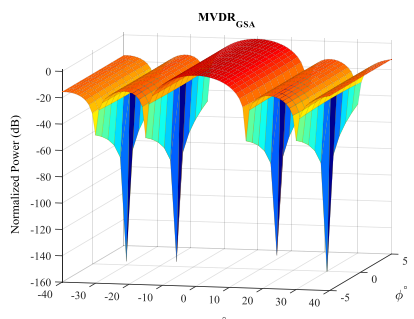

(f)

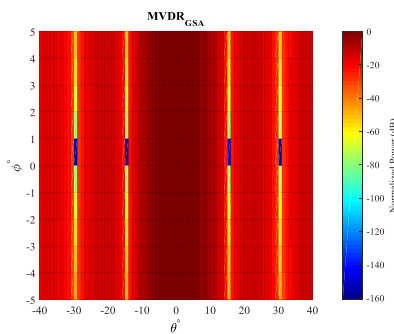

(g)

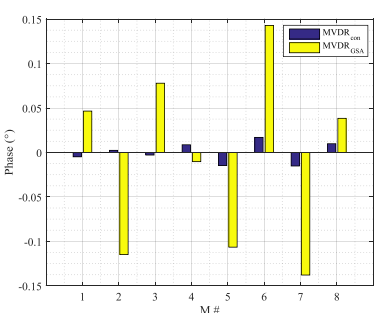

(h)

Fig. 2. Comparison of SINR $\left.\right|_{\max }$ results $\mathrm{MVDR}_{\mathrm{con}} \mathrm{VS}$. MVDR $\mathrm{GSA}_{\mathrm{GS}}$, UOI at $0^{\circ}$, UNOIs at $\pm 15^{\circ}$ and $\pm 30^{\circ}, M=8, t_{\max }=500, N=10$; (a) Best and mean SINR. (b) Normalized beampattern. (c)-(g) $3 \mathrm{D}$ beampattern in term of azimuth and elevation scan angles. (e)-(h) complex weights vector $(A \forall \Phi)$.

Lastly, a third experiment is run to investigate the possibility of having a range of population size and iterations number. Once again, the $\mathrm{MVDR}_{\mathrm{con}}$ and the $\mathrm{MVDR}_{\mathrm{GSA}}$ algorithms are tested with the same simulation setting used in the previous scenario. This scenario is useful to predict the overall performance of the $\mathrm{MVDR}_{\mathrm{GSA}}$, as often the computational effort happens when evaluating the $f f$. Thus, reducing the number of evaluations, while obtaining an acceptable result is important to solve a particular antenna array problem. Fig 3 show a closer look at the MVDR $_{\mathrm{GSA}}$ for optimizing SINR at $N=10,25$ and 50 with each process three different simulation runs using: $100^{\text {th }}, 250^{\text {th }}$ and $500^{\text {th }}$ iterations are defined. According to the results shown in Fig 3 , the increases in the number of iterations has led to increases in the value of SINR owing to the increasing probability of finding better solutions within the search space. It can be observed that for a small number of searching iterations limits the quest for the best solution, for instance at $N=10, t_{\max }=100^{\text {th }}$, obtained mean SINR to be $35.5 \mathrm{~dB}$ ( $29 \%$ decrease) over 20 simulated runs. On the other hand, at $N=25$ with $t_{\max }=100^{\text {th }}$, found mean SINR to be $147.4 \mathrm{~dB}$ give $\approx 193 \%$ improvement compared to $\mathrm{MVDR}_{\text {con. }}$. Conversely, it can also observe that for a number of agents $\left(N=25\right.$ and 50) with a large number iterations $t_{\max }=500^{\text {th }}$ is a slight change in the resultant SINR. Moreover, this improvement is because increasing $N$ can point to increase the likelihood to find the complex excitation coefficient perfectly. Nevertheless, the reduction of $N$ and $t_{\max }$ will lead to a decreased power consumption and the computational time.

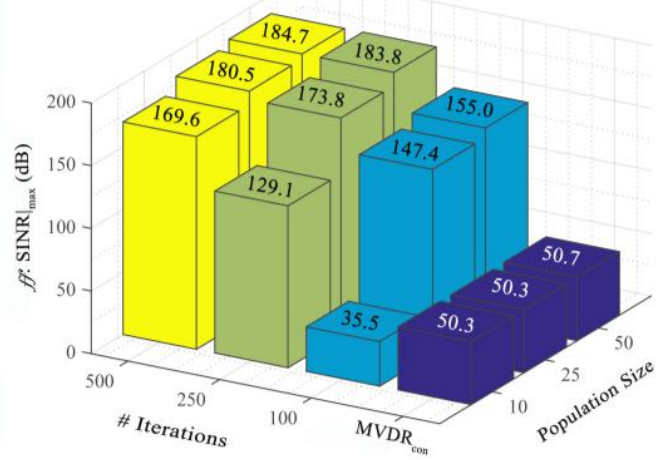

Fig. 3. Performance comparison of average SINR for $M_{V D R}{ }_{G S A}$ 


\section{Conclusion}

Maximizing the SINR is an evaluation criterion that applied to improving the overall desired target signal and simultaneously minimizing the effects of co-channel and mu-ltiple access interference sources. The aim is to use the SINR in the fitness function of the GSA to find a set of array weights. This work has been to investigate the combina-tion of GSA metaheuristic optimization with MVDR technique applied for mitigating MAI sources in adaptive beamforming application. This investigation is carried out by analyzing and comparing the performance based on two figure of merit, SINR and beampattern accuracy for two scan angles. The results showed a better progress in terms of the number of iterations as well as consistency in the values of fitness functi-ons. It is also found that the GSA algorithm achieved deep and sharp null-forming levels compared to conventional MVDR and forming the beampattern accurately. Fu-rther analysis showed that the null width in the elevation angle $\leq 3^{\circ}$ using the proposed $\mathrm{MVDR}_{\mathrm{GSA}}$ method. From the findings, it can be concluded that the metaheuristic optimization has enabled the GSA algorithm to achieve better performance in terms of convergence time and accuracy which can be very helpful in real-time systems. It is recommended that further research should be undertaken to reduce the sidelobe level or the mainlobe beamwidth by using multiobjective fitness function.

\section{References}

1. Capon, J.: High-resolution frequency-wavenumber spectrum analysis. Proceedings of the IEEE 57, 1408-1418 (1969)

2. Van Trees, H.L.: Optimum array processing: part IV of detection, estimation, and modulation theory. Wiley, New York (2002)

3. Gross, F.: Smart antennas with matlab: principles and applications in wireless communication. McGraw-Hill Professional (2015)

4. Godara, L.C.: Application of antenna arrays to mobile communications. II. Beam-forming and direction-of-arrival considerations. Proceedings of the IEEE 85, 1195-1245 (1997)

5. Pan, C., Chen, J., Benesty, J.: Performance study of the MVDR beamformer as a function of the source incidence angle. IEEE/ACM Transactions on Audio, Speech, and Language Processing 22, 67-79 (2014)

6. Darzi, S., Tiong, S.K., Islam, M.T., Ismail, M., Kibria, S.: Optimal null steering of minimum variance distortionless response adaptive beamforming using particle swarm optimization and gravitational search algorithm. In: IEEE 2nd International Symposium on Telecommunication Technologies (ISTT), pp. 230-235. IEEE, (Year)

7. Guney, K., Durmus, A.: Pattern Nulling of Linear Antenna Arrays Using Backtracking Search Optimization Algorithm. International Journal of Antennas and Propagation 2015,(2015)

8. Darzi, S., Sieh Kiong, T., Tariqul Islam, M., Ismail, M., Kibria, S., Salem, B.: Null Steering of Adaptive Beamforming Using Linear Constraint Minimum Variance Assisted by Particle Swarm Optimization, Dynamic Mutated Artificial Immune System, and Gravitational Search Algorithm. The Scientific World Journal 2014, (2014)

9. Ghadian, M., Jabbarian-Jahromi, M., Kahaei, M.: Recursive Sparsity-based MVDR Algorithm for Interference Cancellation in Sensor Arrays. IETE Journal of Research 1-9 (2015) 
10. Chen, Y.L., Lee, J.-H.: Finite data performance analysis of LCMV antenna array beamformers with and without signal blocking. Progress In Electromagnetics Research 130, 281-317 (2012)

11. Besson, O., Vincent, F.E.: Performance analysis of beamformers using generalized loading of the covariance matrix in the presence of random steering vector errors. Signal Processing, IEEE Transactions on 53, 452-459 (2005)

12. http://www.skmm.gov.my/skmmgovmy/media/General/pdf/

13. Feldman, D.D., Griffiths, L.J.: A projection approach for robust adaptive beamforming. Signal Processing, IEEE Transactions on 42, 867-876 (1994)

14. Zaharis, Z.D., Yioultsis, T.V.: A novel adaptive beamforming technique applied on linear antenna arrays using adaptive mutated boolean PSO. Progress In Electromagnetics Research 117, 165-179 (2011)

15. Liu, F., Wang, J., Sun, C., Du, R.: Robust MVDR beamformer for nulling level control via multiparametric quadratic programming. Progress In Electromagnetics Research C 20, 239-254 (2011)

16. Salem, S., Kiong, T.S., Paw, J.K.S., Hock, G.C.: Artificial immune system assisted Minimum Variance Distortionless Response beamforming technique for adaptive antenna system. In: International Conference on ICT Convergence (ICTC'13), pp. 938-943. IEEE,(Year)

17. Chatterjee, A., Mahanti, G.K., Mahapatra, P.R.S.: Design of fully digital controlled reconfigurable dual-beam concentric ring array antenna using gravitational search algorithm. Progress In Electromagnetics Research C 18, 59-72 (2011)

18. Magdy, A., Mahmoud, K., Abdel-Gawad, S., Ibrahim, I.: Direction of Arrival Estimation Based on Maximum Likelihood Criteria Using Gravitational Search Algorithm. PIERS Proceedings 1162-1167 (2013)

19. Shahab, S.N., Zainun, A.R., Mohamed, I.I., Noordin, N.H.: The Impact of Noise Label on Beampattern and SINR of MVDR Beamformer. International Journal of Computing, Communication and Instrumentation Engineering (IJCCIE) 3, 89-95 (2016)

20. Shahab, S.N., Zainun, A.R., Noordin, N.H., Mohamad, A.J.: Performance Analysis of Smart Antenna Based on MVDR Beamformer Using Rectangular Antenna Array. ARPN Journal of Engineering and Applied Sciences 17132-17138 (2015)

21. Souden, M., Benesty, J., Affes, S.: A study of the LCMV and MVDR noise reduction filters. IEEE Transactions on Signal Processing 58, 4925-4935 (2010)

22. Balanis, C.A., Ioannides, P.I.: Introduction to smart antennas. Morgan and Claypool Publishers, Arizona, USA (2007)

23. El Zooghby, A.: Smart antenna engineering. Artech House, Inc., Norwood, MA, USA (2005)

24. Rashedi, E., Nezamabadi-Pour, H., Saryazdi, S.: GSA: a gravitational search algorithm. Information sciences 179, 2232-2248 (2009) 